\section{AB0068 ANALGESIC EFFICACY AND PHARMACOKINETIC AND PHARMACODYNAMIC BASES OF DICLOFENAC CHOLESTYRAMINE, AN IMPROVED DICLOFENAC FORMULATION}

${ }^{1} \mathrm{G}$ Castañeda-Hernandez, ${ }^{2} \mathrm{BE}$ Zazueta-Montiel, 'V Granados-Soto, ${ }^{1} \mathrm{JE}$ Torres-Lopez, ${ }^{1} \mathrm{JT}$ Perez-Urizar, ${ }^{3} \mathrm{R}$ Rosas-Ramos. ${ }^{1}$ Pharmacology, CINVESTAV-IPN, Mexico, Mexico; ${ }^{2}$ Rheumatology, Unidad Reumatologica Y Rehabilitacion Integral, Mexicali, BC, Mexico; ${ }^{3}$ Rheuma/Trauma, Novartis Pharmaceutical, Mexico, Mexico

\subsection{6/annrheumdis-2001.1129}

Background It has been assumed that the analgesic and antiinflammatory effects of diclofenac (DIC) are directly related to drug blood concentrations and, therefore, the time course of the intensity of the response is parallel to that of circulating drug levels. There is evidence, however, that DIC effects last for several hours even if the drug is no longer detectable in the circulation. ${ }^{1}$ It has also been suggested its analgesic action explained in part, by a peripheral mechanism. ${ }^{2}$ If such is the case, DIC response depends on its concentration at the injury site, rather than in plasma. Therefore, a formulation yielding a quick-slow release should show an improved efficacy, as it will allow a rapid drug transfer to the effect compartment, as well as a prolonged presence of DIC in its site of action. ${ }^{3}$

Objectives The purpose of this work was to examine the analgesic efficacy of diclofenac-cholestyramine (DC) a formulation with a quick-slow release profile, ${ }^{4}$ compared to diclofenacsodium (DS) in patients with osteoarthrosis (OA). Additionally, we further investigated the pharmacokinetics (PK) and pharmacodynamics (PD) of DIC in rats, after systemic and local administration, to yield a rational basis to the clinical observations.

Methods The analgesic effect of DC (b.i.d.) and DS $(50 \mathrm{mg}$ t.i. d.) was assayed in 126 patients with OA under a randomised double-blind design. Pain intensity was estimated by means of a visual analogue scale. The PK-PD relationship for DIC was deter-mined in rats using the pain-induced functional impairment model $^{5}$ by the effect compartment approach. ${ }^{3}$ The local antinociceptive effect (ANE) of DIC was studied in rats injured by a plantar injection of $1 \%$ formalin in a hind paw, ${ }^{6}$ and direct drug injection at the injury.

Results Both treatments exhibited a similar analgesic efficacy after 12 weeks. This was observed despite the fact that DS was given t.i.d. while DC was given b.i.d. at a lower daily dose. No significant differences in tolerability were detected. Therefore, clinical results strongly suggest that DC exhibits an improved PK-PD profile. Such assumption was supported by animal studies. Local administration of DIC in formalin-injured rats resulted in a significant ANE compared to saline. This effect was restricted to the local level, as drug injection in the contralateral paw was ineffective. PK-PD modelling in the rat showed that there is no direct link between DIC ANE and its blood concentrations. However, the effect could be related to drug effect-site concentrations, being consistent with the observation of a local effect. Computer gene-rated simulations showed that a quickslow release profile, such as that of DC, allows an optimised DIC transfer to the effect compartment and a prolonged response.

Conclusion DC, a formulation with a quick-slow release profile, exhibits good clinical efficacy and tolerability. PK-PD modelling shows that the quick-slow release profile optimises DIC transfer to its site of action and thus improves the pharmacological response.

\section{REFERENCES}

1 Menassé R, et al. Scand J Rheumatol. 1978;22(Suppl 5)

2 Tonussi CR, Ferreira SH. Eur J Pharmacol. 1994;251:173

3 Colburn WA. J Pharmacokinet Biopharm. 1981;9:367

4 Kurowski M, et al. Int J Clin Pharmacol Ther. 1994;32:433

5 Granados-Soto, et al. J Pharm Pharmacol. 1992;44:741

6 Islas-Cadena, et al. J Pharmacol Toxicol Methods 1999;42:87

\section{FRI0001 FOLINIC ACID SUPPLEMENTATION REVERSES MACROCYTOSIS WITHOUT ALTERING EFFICACY IN PATIENTS WITH ARTHRITIS TREATED WITH METHOTREXATE}

B Mukerji. Internal Medicine, SIU School of Medicine, Springfield, USA

\subsection{6/annrheumdis-2001.1130}

Background Methotrexate is known to cause folate deficiency which can be manifested early on as macrocytosis in a complete blood count. Some clinical manifestations of folate deficiency such as cytopenia, anorexia, stomatitis, and gastrointestinal intolerance are also observed during low dose methotrexate (MTX) treatment in various forms of arthritis.

Objectives The purpose of this study was to determine the effectiveness of daily folinic acid supplementation in reversing macrocytosis noted in some patients with rheumatoid arthritis (RA) and psoriatric arthritis (PsA) treated with low dose methotrexate and folic acid and to see if it altered the disease modifying activity of the drug.

Methods Twenty-eight patients were studied. There were 20 women and eight men with an age range of 34-82 years. Twenty-four patients had RA and four had PsA. They were all treated with low dose MTX between 5-15 mg. weekly and folic acid two mg. orally daily, as well as a nonsteroidal antiinflammatory drug and in some patients low dose oral prednisone. Additionally, 16 patients took hydroxychloroquine sulfate, eight patients sulfasalazine, and two patients auranofin. All patients had normal thyroid function. Twenty-three patients (82\%) developed macrocytosis after an interval of three to four years on MTX and folic acid. A favourable response was noted in two patients by reversal of their macrocytosis when their daily folic acid dose was increased to three mg. For 21 patients the folic acid was discontinued and supplemented orally with five mg. folinic acid daily. Of the five patients who did not develop macrocytosis, four took weekly MTX of seven and a half $\mathrm{mg}$. or less. One took $15 \mathrm{mg}$. MTX weekly but also received intramuscular testosterone every three weeks for hypogonadism.

Results Patients were followed for three to seven years. Of the 21 patients on folinic acid supplementation, 20 (95\%) had a reversal of their macrocytosis in 12-15 months. There was no exacerbation of their underlying arthritis. This was determined by joint count, tenderness, limitation of motion and flexion contractures of affected joints. When the folinic acid was discontinued and folic acid reinstituted, macrocytosis recurred in two to three years in five patients and responded to another course of folinic acid. Eight patients who had active disease from the onset continued to have active disease and six were subsequently given etanercept injections. The remaining 13 patients continued to show improvement of all parameters of disease activity. The one non-respondent had active PsA.

Conclusion These observations suggest that reversal of macrocytosis can be accomplished with daily use of five mg. folinic acid without diminishing the effectiveness of low dose MTX in patients with RA and PsA. 